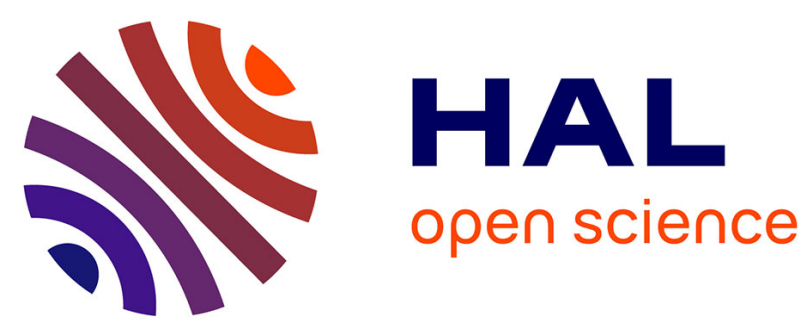

\title{
Assembly and Characterizations of Bifunctional Fluorescent and Magnetic Microneedles Displaying Length Tunability over One Decade
}

Jean-Baptiste Lugagne, Gwennhaël Brackx, Emek Seyrek, Sophie Nowak, Yann Sivry, Letícia Vitorazi, Jean-François Berret, Pascal Hersen, Gaëlle

Charron

\section{To cite this version:}

Jean-Baptiste Lugagne, Gwennhaël Brackx, Emek Seyrek, Sophie Nowak, Yann Sivry, et al.. Assembly and Characterizations of Bifunctional Fluorescent and Magnetic Microneedles Displaying Length Tunability over One Decade. Advanced Functional Materials, 2017, 27 (31), pp.1700362. 10.1002/adfm.201700362 . hal-02342490

\section{HAL Id: hal-02342490 \\ https://hal.science/hal-02342490}

Submitted on 16 Dec 2019

HAL is a multi-disciplinary open access archive for the deposit and dissemination of scientific research documents, whether they are published or not. The documents may come from teaching and research institutions in France or abroad, or from public or private research centers.
L'archive ouverte pluridisciplinaire HAL, est destinée au dépôt et à la diffusion de documents scientifiques de niveau recherche, publiés ou non, émanant des établissements d'enseignement et de recherche français ou étrangers, des laboratoires publics ou privés. 


\title{
Assembly and Characterizations of Bifunctional Fluorescent and Magnetic Microneedles Displaying
}

\section{Length Tunability over One Decade}

Jean-Baptiste Lugagne ${ }^{\mathrm{a}}$, Gwennhaël Brackxª ${ }^{\mathrm{a}}$ Emek Seyrek ${ }^{\mathrm{a}}$, Sophie Nowak $^{\mathrm{b}}$, Yann Sivry ${ }^{\mathrm{c}}$, Leticia Vitorazi ${ }^{\mathrm{a}}$, Jean-François Berret ${ }^{\mathrm{a}}$, Pascal Hersen $^{\mathrm{a}}$ and Gaëlle Charron *a

${ }^{a}$ Laboratoire Matière et Systèmes Complexes, UMR 7057, Université Paris Diderot, Paris, France

bTODYS, UMR 7086, Université Paris Diderot, Paris, France 'IPGP, UMR 7154, Université Paris Diderot, Sorbonne Paris Cité, CNRS, Paris, France

December 21, 2016

\begin{abstract}
We report on the fabrication of bifunctional magnetic and fluorescent microneedles $(\mu \mathrm{NDs})$ made of a ternary mixture of magnetic nanoparticles (NPs), quantum dots (QDs) and polyelectrolyte. The assembly relies on the electrostatic complexation of negatively charged NPs with positively charged polymer strands and is controlled by the charge ratio between the nanoparticulate building blocks and the polymer mortar. We show that the resulting $\mu$ NDs retain the fluorescent and superparamagnetic

*gaelle.charron@univ-paris-diderot.fr
\end{abstract}


properties of their NP constituents. Moreover, using a combination of core and surface characterisations and a state-of-the-art image analysis algorithm, we thoroughly investigate the dependence of the brightness and length on the ternary composition. In particular, based on statistics on hundreds of $\mu$ NDs with a range of compositions, we show that the length can be robustly tuned over one decade to match the relevant length scales of various applications.

Keywords: microneedles, magnetic nanoparticles, quantum dots, nanoparticle assembly, confocal fluorescence microscopy

\section{Introduction}

1D-magnetic microstructures made of single magnetic microparticles or assemblies of magnetic nanoparticles can find applications in a wide variety of fields that includes composite materials, microfluidics and micro-mechanics. Micro-rods of non-magnetic material coated with iron oxide nanoparticles have been used to mechanically reinforce polymer matrices through applying small magnetic fields [1]. Micro-machined pieces of magnetic materials or chains of magnetic colloids have been applied as micro-stir bars to increase the efficiency of mixing in microfluidic channels and micro-droplets [2-6]. Mixing at the microscale can also be enhanced using micropillars or artificial cilia consisting of magnetic micro or nanoparticle assemblies that can be actuated through application of a rotating or a swinging magnetic field [7]. These magnetic cilia can form the basis of flow or vibration sensing when coupled to a giant magnetoresistive sensing element that monitors their deflection [8,9]. Alternatively, the bending of these cilia under a static external field can serve as a mean to apply controlled stresses to soft materials deposited on the substrate such as tissues, films or cells in order to study their mechanical responses [10-12]. In the same line, free standing magnetic micro-rods can be used as active micro-rheometers to probe the viscoelastic properties of complex fluids, including living cells, with microscale resolution [13, 14].

Many of these applications would benefit from the addition of a fluorescent functionality to the magnetic one. Incorporating fluorophores into the 1D-microstructures enables easier imaging in scattering media. It could allow for optical bar-coding of the magnetic micro- 
actuators. In cell mechanics studies, it could give insights into the environment of the magnetic probe through colocalisation experiments involving organelle-specific fluorophores. Moreover, most of these applications simultaneously require the length of the 1D-magnetic microstructures to be adjusted to the problem at play. Micro-stirrers need to be attuned to the dimensions of the fluid compartment and to its viscosity. The range of forces to which magnetic microposts are sensitive depends on their length and thickness. To be able to resolve inhomogeneities in viscoelastic properties at the microscale, magnetic microrheometers need to be of dimensions comparable to the typical length scale of the structural inhomogeneities $[15,16]$.

Bifunctional magnetic and fluorescent nano or microspheres have been reported in the past. They were developed mostly towards applications in bimodal magnetic resonance and fluorescence imaging, drug delivery or bioassays.

These materials have been synthesised according to various strategies: core-shell particles, where a core of magnetic material (e.g. iron oxide nanoparticles) is encased in a shell of fluorescent one (e.g. dye-labelled silica or quantum dots) or the other way round [17-19]; raspberry-like particles, where a large particles of one material are decorated on their surface with smaller particles of the other material [20,21]; colloidosomes self-assembled from fluorescent and magnetic colloids [22]; beads in which particles of both materials have been coprecipitated or co-embedded in a silica or polymer matrix [23-25]; and particles made of oxides of rare earth ions [26]. Most of these strategies lead to bifunctional spheres in the 0.1 to $1 \mu \mathrm{m}$ range whose size cannot be easily tuned. In general, they are not amenable to synthesize 1D structures. Moreover, in many cases the synthetic cost is high, either because of the minute scale of production or to the time or number of steps required. We report here on the fabrication of bifunctional magnetic and fluorescent 1D-structures of micrometric length assembled by controlled aggregation of a ternary mixture of polyelectrolyte, superparamagnetic nanoparticles and quantum dots. The building blocks are simple and easily sourced. Assembly proceeds in water, in less than half an hour, to give rise to wire or needle-like aggregates of millions of nanoparticles that are responsive under an external magnetic field and can be imaged by conventional epifluorescence microscopy. Interestingly, by playing on the ternary composition, their length can be tuned in the 5-50 $\mu \mathrm{m}$ range to match the needs 
of many microfluidic or biophysical applications. As an illustration of the potential of the microneedles $(\mu \mathrm{NDs})$ for cell mechanics studies, we show that they are readily internalised by HeLa cells and that their fluorescence can be used to assess their localisation with regards to cellular compartments.

\section{Results and discussion}

\subsection{General strategy for assembling fluorescent magnetic micronee- dles}

\subsubsection{Assembly of 1st generation $\mu$ NDs}

The bifunctional magnetic $\mu$ NDs derive from a first generation of magnetic-only 1D-microstructures reported previously. This first generation of magnetic $\mu$ NDs was obtained by electrostatic complexation of negatively charged magnetic nanoparticles (mNPs) with polycationic polymers in a kinetically controlled fashion [27-29]. The magnetic building blocks consisted of 6-8 $\mathrm{nm}$ superparamagnetic $\mathrm{Fe}_{2} \mathrm{O}_{3}$ maghemite NPs coated in a $3 \mathrm{~nm}$-thick layer of sodium polyacrylate that imparted them a persistent colloidal stability, even at high ionic strength $(\geq 1 \mathrm{M})$. Various polyelectrolytes bearing positive charges through ammoniums (either quaternary or proton-exchanging) were investigated and all lead to successful assembly [30].

Due to the strong driving force for electrostatic assembly, direct mixing of the NP building blocks and polyelectrolyte glue resulted in macroscopic precipitation of large aggregates. The assembly protocol was therefore designed to slow down the interaction. To this end, the constituents were mixed in the presence of a screening electrolyte at high ionic strength (typically in the M range), at which stage they remained individually dispersed. Assembly was then driven by slow dialysis of the screening electrolyte to give rise to spherical clusters. When performing the assembly under a static magnetic field (typically of $0.3 \mathrm{~T}$ ), 1D structures grew and lead to microneedles between 150 and $400 \mathrm{~nm}$ in width and 1 to $500 \mu \mathrm{m}$ in length that displayed superparamagnetism inherited from their iron oxide constituents. Starting binary compositions were referenced using the charge ratio $Z$, defined as the number of negative charges brought by the mNPs divided by the number of positive charges brought 
by the polymer (1).

$$
Z=\frac{C_{m N P}^{\ominus} V_{m N P}}{C_{p o l}^{\oplus} V_{p o l}}
$$

\subsubsection{Choice of constituents for fluorescent magnetic $\mu$ NDs}

One possible strategy to incorporate a fluorescent functionality into the magnetic $\mu$ NDs described above consists in substituting fluorescent building blocks for a fraction of the magnetic ones. Fluorescent NPs then need to present the same chemical reactivity as mNPs with regards to the assembly mechanism. This implies that they must bear negative charges on their surface and, critically, display colloidal stability even at high ionic strength. With the aim of tracking the $\mu$ NDs using fluorescence (video) microscopy, the $\mu$ NDs should ideally be as bright as possible. Indeed, strong brightness not only increases contrast but also enables the use of short exposure times and therefore give access to shorter timescales of dynamic tracking. Moreover, the $\mu$ NDs should display homogeneous fluorescence, , namely should be homogeneously doped with fluorescent blocks, so that the fluorescence profile tightly mirrors the shape of the object. In a solid state solution, doping is facilitated by a similarity of size between the dopant and the host. Based on the same principle, the fluorescent building blocks and the magnetic ones should have diameters of the same order of magnitude.

Taking into account these criteria, we selected the $\mathrm{AgInS}_{2}-\mathrm{ZnS}$ (ZAIS) quantum dots (QDs) described by Regulacio et al. [31] as fluorescent building blocks (Fig. 1). These hydrosoluble QDs were synthesised directly in water and were a few $\mathrm{nm}$ in diameter. Their emission was composition-dependent and could be tuned from 525 to $640 \mathrm{~nm}$, with a maximum reported quantum yield of $20 \%$. Interestingly, these QDs were synthesized in the presence of polyacrylic acid, which acted as a capping agent. Having a diameter within the same range as the mNPs and the exact same coating, we expected these QDs to be an ideal dopant for the $\mu$ NDs. 

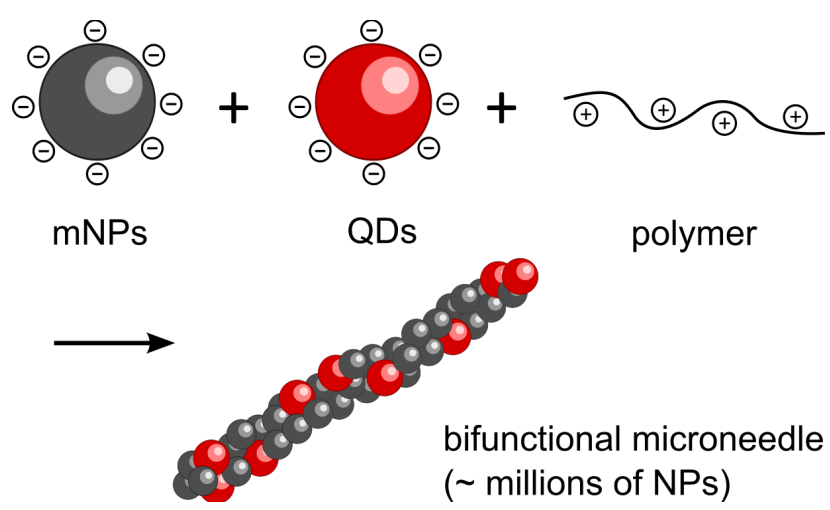

Figure 1: Synthetic scheme for assembly of bifunctional fluorescent magnetic $\mu$ NDs

\begin{tabular}{cccccc}
\hline NPs & $D_{c}(\mathrm{~nm})$ & $D_{H}(\mathrm{~nm})$ & core & coating & charge density $(\mathrm{mmol} / \mathrm{g})$ \\
\hline QDs & $5.8 \pm 1.2$ & $\sim 13$ & $\mathrm{Ag}_{0.23} \mathrm{In}_{0.58} \mathrm{Zn}_{1.54} \mathrm{~S}_{3}$ & PAA & $3.865 \pm 0.242$ \\
\hline mNPs & $9.4 \pm 1.7$ & $\sim 31$ & $\mathrm{Fe}_{2} \mathrm{O}_{3}$ & PAA & $0.975 \pm 0.262$ \\
\hline
\end{tabular}

Table 1: Summary of QD and mNP characteristics

\subsubsection{Characterisations of magnetic and fluorescent building blocks for NW assembly}

The mNPs used for $\mu$ ND assembly were synthesised following the Massart method and coated in a post-synthetic fashion with PAA, as described earlier [32,33]. The detailed characterizations of the exact batch used in this study are presented in ESI. Hereafter, we present the characterisations of the QDs we synthesized using the procedure from Regulacio et al. with few modifications, and compare the QD characteristics to those of the mNPs used for $\mu$ ND assembly (Table 1) [31].

Optical properties. Iron oxide NPs present an intense and continuous absorption in the UV-vis range that vanishes close to $610 \mathrm{~nm}$. We therefore aimed at synthesizing orange to red-emitting QDs to prevent fluorescence quenching through energy transfer to or reabsorption by the host matrix of the $\mu \mathrm{ND}$. The synthesized QDs had a maximum emission peak centered at $600 \mathrm{~nm}$ and a moderate quantum yield of $7 \%$, which was compensated by the emission sitting well outside the iron oxide absorption range (Figure 2). According to Inductively Coupled Plasma Optical Emission Spectrometry (ICP-OES) analysis, the 


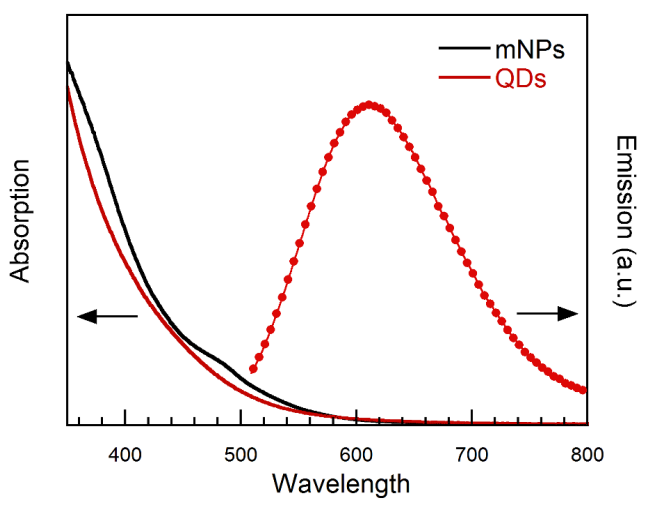

Figure 2: Absorption spectra of mNPs and QDs (plain) and emission spectrum of QDs under $490 \mathrm{~nm}$ irradiation (dots).

QDs had a non-stoechiometric $\mathrm{Ag}_{0.23} \mathrm{In}_{0.58} \mathrm{Zn}_{1.54} \mathrm{~S}_{3}$ formula, reflecting an excess chalcogen compared to the metal ions and a defect in silver compared to indium, which is consistent with orange emission (Table S1) [34].

Size properties. TEM images of QDs reveal a $5.8 \pm 1.2 \mathrm{~nm}$ core diameter based on counting 175 NPs (Fig. S1). The hydrodynamic diameter as determined by DLS was 13 nm in pure water at $\mathrm{pH} 8.5$ (Fig. S2). The QDs and mNPs therefore had dimensions of the same order of magnitude, both regarding their inorganic core and their size in solution. Importantly, the hydrodynamic diameter of QDs remained unchanged in the screening electrolyte solution used for $\mu \mathrm{ND}$ assembly $\left(1 \mathrm{M} \mathrm{NH} \mathrm{NH}_{4} \mathrm{Cl} / \mathrm{NH}_{4} \mathrm{OH}\right)$ over a period of days, evidencing remarkable colloidal stability due to the PAA coating. Just as the mNPs, the QDs were resilient towards the NW assembly conditions.

Charge density and concentration. As evidenced by gel electrophoresis on agarose and consistently with PAA coating, the QDs were negatively charged (Fig. S9). They also exhibited a higher number of surface carboxylates per core mass unit than the mNPs, a fact that was confirmed by analysis of the composition of both batches of QDs and mNPs, by ICP-OES for the inorganic core elemental concentrations and $\mathrm{pH}$-titration for the surface carboxylates (see ESI) [35]. Detailed compositions of the stock solutions are provided in Tables S1 \& S5. 


\subsection{Assembly of fluorescent magnetic microneedles}

Assembly of fluorescent magnetic $\mu$ NDs was performed following the same synthetic procedure as those developped previously for magnetic-only $\mu$ NDs, but for the substitution of QDs for a fraction $w$ of mNPs (Eq. (2)). For a given assembly procedure, the starting reagent stoechiometry is unambiguously defined by two synthetic parameters: $w$ and the previously defined $Z$ ratio between negative charges brought by the nanoparticles and positive charges brought by the polymer, which now takes into account the contribution of QDs

(Eq. (3)). Alternatively, the ternary composition can be depicted using the molar fractions of the charges respectively brought by QDs, mNPs and polymer, according to Equations (4). A set of $15\{Z ; w\}$ compositions with $Z \in[0.62-7.01]$ and $w \in[0-0.2]$ was investigated (Table S6). The individually dispersed mNPs are too small to be collected using a table magnet but their aggregates have a much higher saturation magnetization. Successful decantation under an applied magnetic field (typically of few hundredth of Tesla) therefore evidences that assembly has taken place. According to this indicator, we observed that all investigated compositions successfully led to $\mu \mathrm{ND}$ assembly.

$$
\begin{gathered}
w=\frac{V_{Q D}}{V_{m N P}+V_{Q D}} \\
Z=\frac{C_{Q D}^{\ominus} V_{Q D}+C_{m N P}^{\ominus} V_{m N P}}{C_{p o l}^{\oplus} V_{p o l}} \\
x_{Q D}=\frac{C_{Q D}^{\ominus}}{C_{Q D}^{\ominus}+C_{m N P}^{\ominus}+C_{p o l}^{\oplus}} \\
x_{m N P}=\frac{C_{m N P}^{\ominus}}{C_{Q D}^{\ominus}+C_{m N P}^{\ominus}+C_{p o l}^{\oplus}} \\
x_{p o l}=\frac{C_{p o l}^{\oplus}}{C_{Q D}^{\ominus}+C_{m N P}^{\ominus}+C_{p o l}^{\oplus}}
\end{gathered}
$$




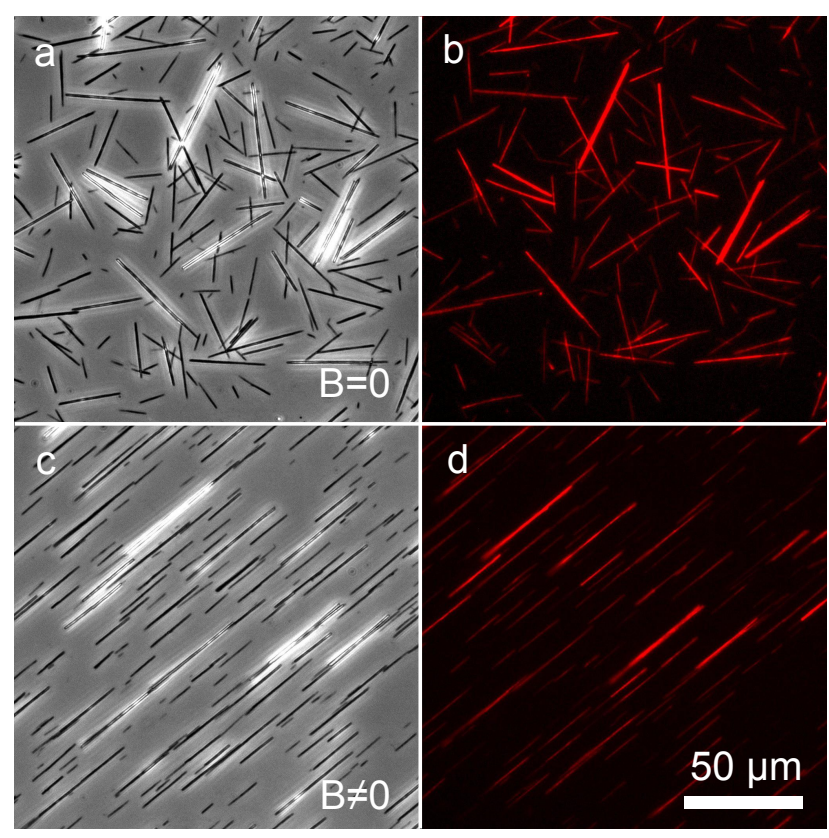

Figure 3: Phase contrast and epifluorescence microscopy images of $\{Z=3.62 ; w=0.15\}$ $\mu$ NDs, without $(\mathrm{a}, \mathrm{b})$ and under $(\mathrm{c}, \mathrm{d})$ static magnetic field.

\section{$2.3 \quad$ Properties of QD-doped magnetic $\mu$ NDs}

\subsubsection{Overview of properties: fluorescence and responsiveness under magnetic actuation}

Figure 3a depicts a phase $20 \mathrm{X}$ contrast image of the aggregates obtained starting from the $\{Z=3.62 ; w=0.15\}$ composition. Straight $\mu \mathrm{ND}$ are obtained with lengths ranging from about 5 to $50 \mu \mathrm{m}$ and diameters of about 0.3-1.0 $\mu \mathrm{m}$ (see ESI for TEM characterisation of width). Their morphology is identical to the magnetic-only $\mu$ ND reported previously. Figure $3 \mathrm{~b}$ displays the same specimen area imaged by epifluorescence microscopy. The $\mu$ ND emit in the red range under green excitation and appear homogeneously bright, evidencing homogeneous incorporation of the QDs in the host $\mu$ ND matrix and retention of their optical properties. When depositing a soft permanent magnet on the microscope stage, the $\mu$ ND respond to the external magnetic actuation by aligning along the field lines (Fig. 3c \& 3d). Upon suppressing the external magnetic field, the $\mu \mathrm{ND}$ relax towards randomized orientations, thereby evidencing superparamagnetism.

Interestingly, the morphology and in particular the length of the $\mu$ NDs vary with the 
starting composition (Fig. 4). While $\{Z=4.20 ; w=0.20\}-\mu$ NDs have a mean length of 13 $\mu \mathrm{m},\{Z=1.40 ; w=0.20\}-\mu$ NDs that have the same QDs substitution ratio are on average 3 times longer $(39 \mu \mathrm{m})$. Both samples display straight persistent needle-like aggregates. In contrast, the $\{Z=1.21 ; w=0.15\}$ sample contains large, soft, hair strand-like bundles of $\mu \mathrm{NDs}$ whose length cannot be systematically determined because of inter- $\mu$ NDs aggregation, along with some individual $\mu$ NDs. In addition, the longer the $\mu$ NDs or bundles of $\mu$ NDs, the brighter they are. As both the length of the $\mu$ NDs and their brightness ultimately determine their potential application, a systematic investigation of these parameters as a function of ternary composition was conducted.

\subsubsection{Systematic investigation of length and brightness as a function of ternary composition}

Tools for image analysis. The distribution of length was analysed from phase contrast microscopy images acquired with 10, 20 and 40X objectives. Lengths were extracted using a custom-written, dedicated image analysis Matlab code The algorithm for $\mu$ ND recognition was based on the linear Hough transform, which is a feature extraction technique devoted to the identification of lines [36]. The software is successful in recognizing single objects in an entangled bunch of $\mu$ NDs but not when the $\mu$ NDs are bundled (Fig. S11). It is biased against smaller $\mu$ NDs due to their comparatively lower contrast. The software features a graphical user interface (GUI) for quick and handy correction of the most obvious errors, such as $\mu$ NDs identified shorter than they are due to uneven contrast along their length or wires aligned tip-to-tip mistaken for one long object. Using this automated image analysis tool, $750 \mu$ NDs on average were counted per sample comprising individual $\mu$ NDs, with no less than $200 \mu$ NDs counted for that having the highest mean length. All length distributions were log-normal with relative standard deviations ranging from 15 to $23 \%$ (Fig. 4 \& S12). Fluorescence analysis was performed based on epifluorescence microscopy images acquired with a single 20X objective. The brightness of each sample was taken as the mean background-corrected intensity of the identified bright pixels in the set of sample images. The dependence of brightness and mean length on ternary composition are depicted in Fig. 5 (see also Table S7 and Fig. S14) 

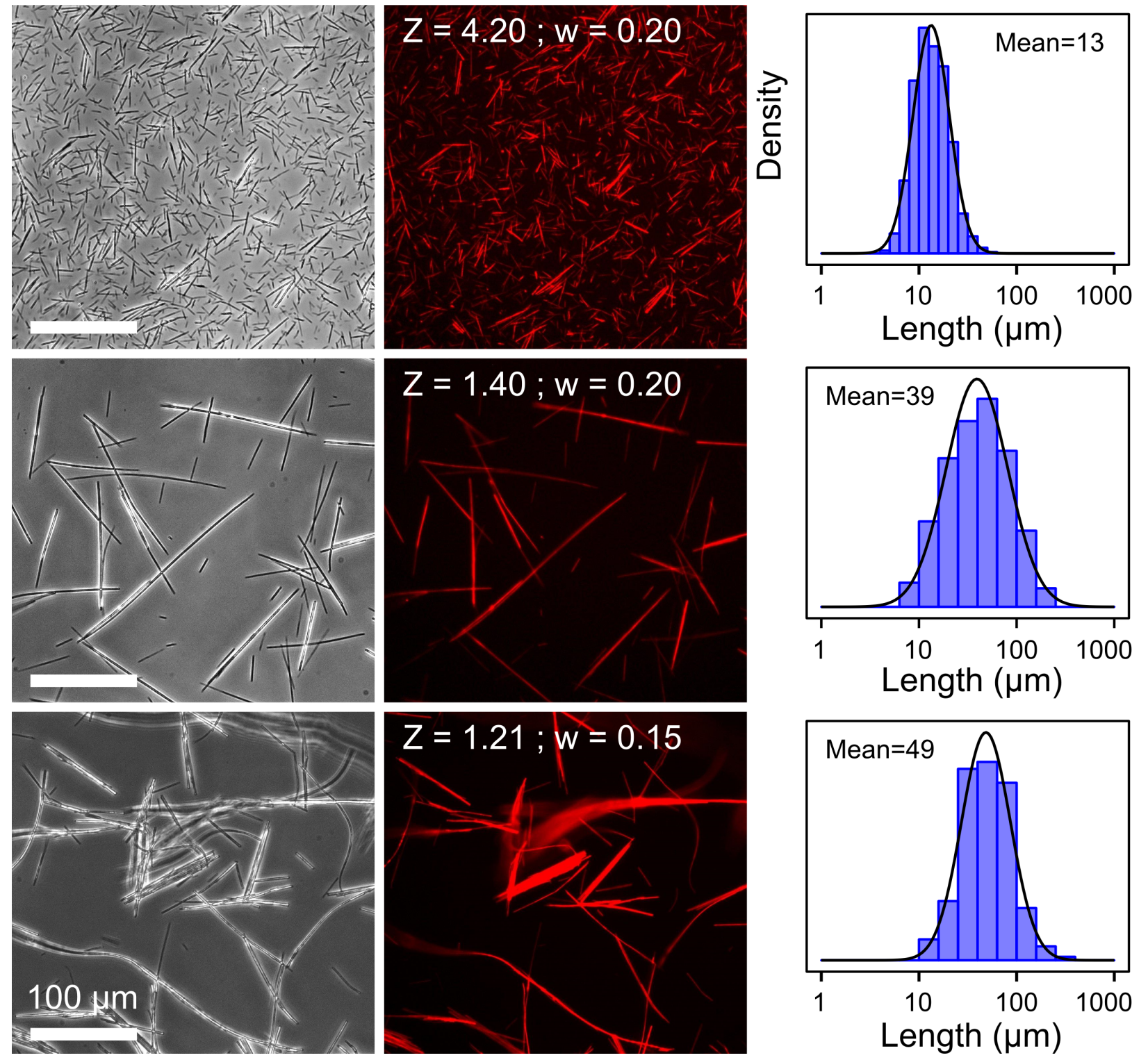

Figure 4: Phase contrast (left), epifluorescence (middle) microscopy images and associated length distributions (right) of bifunctional $\mu$ NDs with various compositions. 

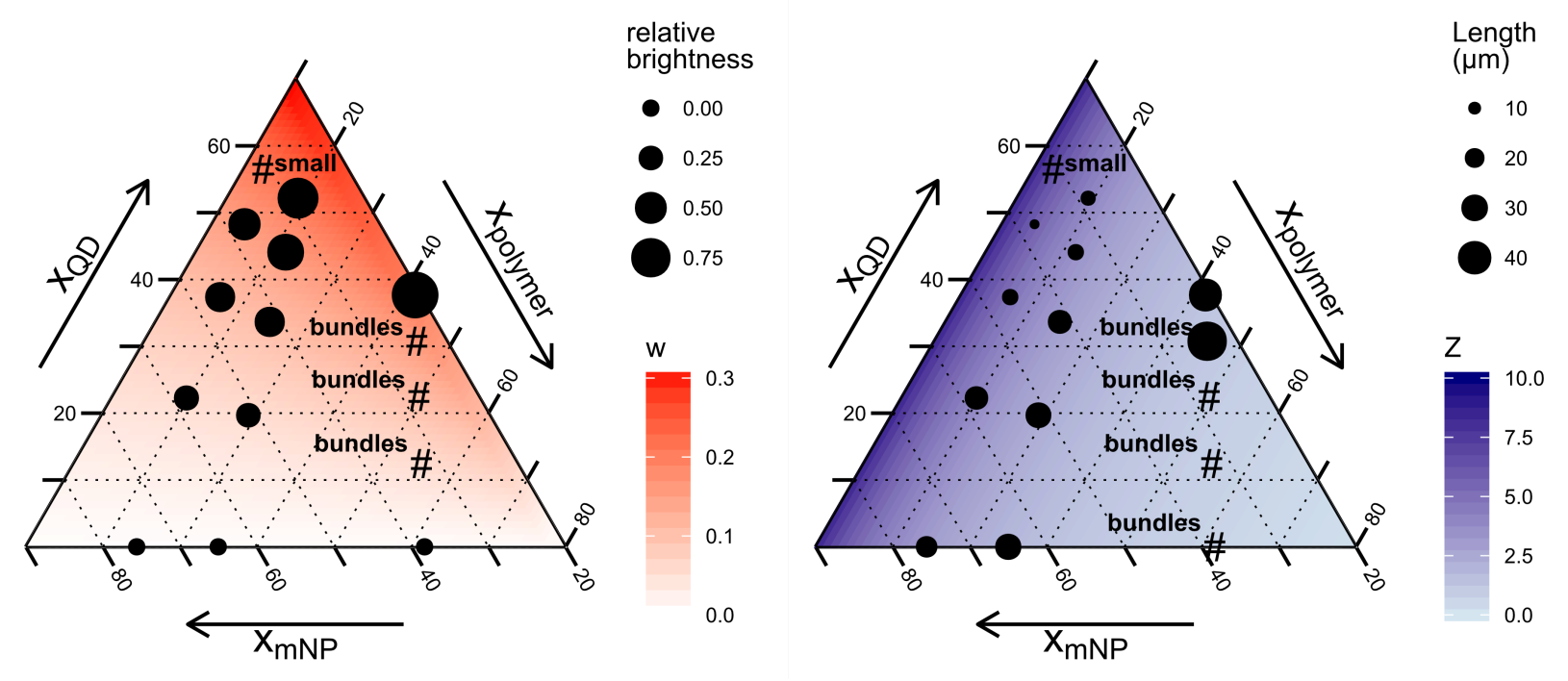

Figure 5: Relative fluorescence brightness (left) and mean length (right) as a function of ternary molar composition.

Tuning of brightness. For convenience, the QD-doping ratio $w$ is mapped over the range of ternary compositions in Figure 5 (left). As a general trend, starting compositions richer in QDs display higher relative brightness. This observation evidences that the actual incorporation ratio parallels the starting doping ratio and supports the hypothesized similarity in reactivity between PAA-coated QDs and PAA-coated mNPs.

Tuning of length. Out of 15 compositions, the mean length of 11 samples could be extracted. Mapping the $Z$ ratio over the ternary diagram reveals that the length decreases with increasing $Z$, with the geometric mean length ranging from 5.7 to $48.5 \mu \mathrm{m}$ for $Z=6.03$ to 1.21 respectively. Three compositions lead to large bundles of aggregated $\mu$ NDs whose length could not be extracted; they corresponded to the weakest $Z$ ratios across the whole range of molar fractions $(Z=0.62,0.82 \& 1.01$ respectively). The last remaining sample corresponded to the highest $Z$ value (7.01). For this sample, the $\mu$ NDs could not be resolved using the 40X objective, although assembly had indeed taken place since the product could be magnetically decanted with a table magnet, albeit admittedly very slowly.

The dependence of the mean length on the composition was rationalised earlier for magnetic-only $\mu$ NDs [28]. The assembly of $\mu$ NDs was shown to occur in two steps that are not separated in time (Fig. S10). In a first nucleation step, supra-colloidal clusters are 
formed due to the electrostatic complexation between negatively charged mNPs and positively charged polymer strands once the ionic strength falls below a certain threshold. In a second growth step, clusters align along the magnetic field lines, assemble and then fuse due to magnetic dipolar attraction (Fig. S10). The surface charge of the clusters was found to mirror the $Z$ ratio of the starting mixture, with compositions featuring excess mNPs $(Z>1)$ leading to negatively charged clusters and those featuring an excess polycation $(Z<1)$ leading to positively-charged ones [30]. The kinetics of assembly therefore results from the balance between dipolar magnetic attraction and electrostatic repulsion between the supra-colloidal clusters. Accordingly, $\mathrm{Z}=1$-magnetic-only $\mu \mathrm{NDs}$, which stem from intermediate clusters having the weakest surface charge, were systematically found to be the largest [30].

Here, we attempted at modelling the dependency of the length on compositional parameters in the case of the bifunctional $\mu$ NDs. The dependence of the length on the $Z$ ratio in fluorescent magnetic $\mu$ NDs appears fully consistent with the afore-mentioned mechanism. There is strong anticorrelation at the $95 \%$ confidence level between length and $Z$ ratio (see ESI for details of the t-test). The shortest $\mu$ NDs correspond to compositions having the largest $Z$ absolute values due to the increased electrostatic repulsion between clusters. As the QD building blocks have a higher surface charge density than the mNPs,these shortest $\mu$ NDs are also the richest in QDs. Since ZAIS QDs are diamagnetic, their incorporation should lessen the saturation magnetisation of the clusters. For a given $Z$ value, a higher QD doping ratio should therefore lead to less intense magnetic dipolar attraction between clusters. One might expect this phenomenon to slow down the assembly and lower the length of the resulting $\mu$ NDs. However, a t-test conducted on the data indicated no significant correlation between the length and the doping ratio $w$ (see ESI). Finally, the dependency of the length on the compositional parameters could be simply described as Equation (5) (Figure 6).

$$
L \approx 65 \times \exp (-0.33 Z)
$$

where $L$ is the geometric mean length and $Z$ varies in the $1.2-6.0$ range (Akaike information criterion was applied to choose the best model, see ESI). 


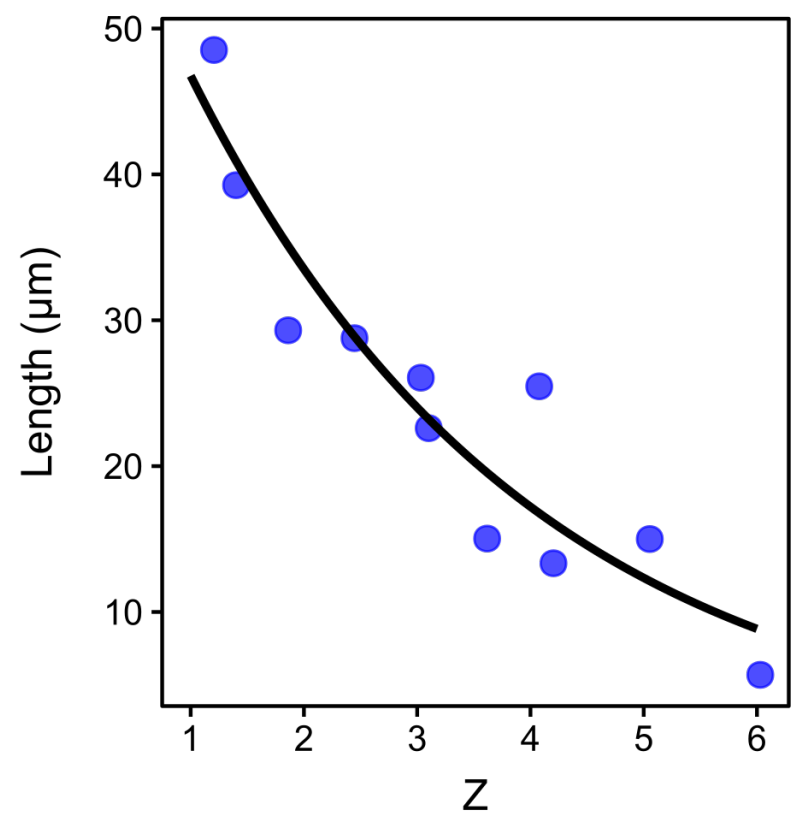

Figure 6: Geometric mean length of $\mu$ NDs as a function of $Z$ (blue dots) and best fit line according to Eq. (5) (black line).

Because of their length tunability in the 5 - $50 \mu \mathrm{m}$ range and the ease with which they can be assembled, the bifunctional fluorescent magnetic $\mu$ NDs could find applications in multiple domains. Microfluidic channels have a typical width of $50-200 \mu \mathrm{m}$. At the upper end of the size window, 30-50 $\mu \mathrm{m} \mu \mathrm{NDs}$ could be used as individual micro-stir bars as a cheaper alternative to micromachinned devices that have been reported earlier [5]. Shorter $\mu$ NDs could be easily incorporated as multiple stir bars in arrays of microdroplets to efficiently churn up the whole solution compartments $[3,4]$. The magnetic actuation and fluorescent features could be exploited in simultaneous magnetic and fluorescence-assisted cell sorting [37]. Alternatively, the bifunctional $\mu$ NDs could act as probes for active viscosimetry-based label-free bioassays [38]. In both of these assaying applications, the synthetic control over the length would enable fine tuning of probe to the application through optimisation of the saturation magnetisation of the $\mu$ NDs. Finally, $\mu$ NDs could allow to probe the mechanical properties of biological materials such as tissues, films, individual cells or subcellular compartments using magnetic actuation-based techniques coupled to fluorescence imaging. Lately, the viscoelastic properties of cells and tissues have attracted much attention in the frame of medical 
research. For instance, the mechanical stiffness of cancer cells has been shown to correlate with their metastatic potential, with softer more deformable cells having the highest migratory potential and invasiveness [39]. Moreover, cell state or fate can be controlled by the viscoelastic properties of its environment [40]. The phenomena at play are difficult to study by conventional macroscopic rheological methods since the samples typically come as tiny specimens and biological fluids are generally complex, ie. they behave differently at different length scales. In this context, the size tunability over the $5-50 \mu \mathrm{m}$ range is especially appealing since it would enable the study of a given material from the scale of assemblies of cells down to the subcellular level with a single technique suited to microscopic specimens. Such multiscale study would shine light on the respective contributions and interplay of the mechanics of cells, organites and surrounding matrix.

\subsection{Intracellular imaging of fluorescent magnetic microneedles}

Since many of these applications would rely on interaction of the bifunctional $\mu$ NDs with cells and subsequent fluorescent imaging, we hereafter examine whether the present bifunctional fluorescent magnetic $\mu$ NDs would be suitable to probe individual cells via confocal fluorescence microscopy. Magnetic-only $\mu$ NDs were previously found to be internalised by mouse fibroblasts and human cancer cells, a phenomenon which was used to extract viscoelastic properties of these cells through rotational magnetic spectroscopy [13]. Here, HeLa were incubated with QD-doped $\mu \mathrm{NDs}$, fixed and stained with DAPI in order to fluorescently label their nucleus. Figure 7 displays bright field (first row) and fluorescence (middle row) confocal images acquired in two focal plans lying $6 \mu \mathrm{m}$ apart. The blue and red fluorescence channels correspond to DAPI and QD emissions, respectively. In the left panel, the nuclei are out of focus while they come into focus in the right panel. On bright field images, a few $\mu \mathrm{ND}$ can be spotted, mostly in the right panel, but their localisation with regards to the cellular compartments is difficult to assess. On fluorescence images (middle row), $\mu$ NDs can be more easily outlined as red dashes. On cell $\mathbf{C} 1$ in left panel, a few $\mu$ NDs appear on top of the out-o-focus nucleus. These $\mu$ NDs disappear when the C1-nucleus comes into focus in the right panel, while many more $\mu$ NDs with sharp contours appear around it. Reconstruction of the Z-profile of cell $\mathbf{C 1}$ (lower row) conveniently shows that $\mu$ NDs are internalised within 


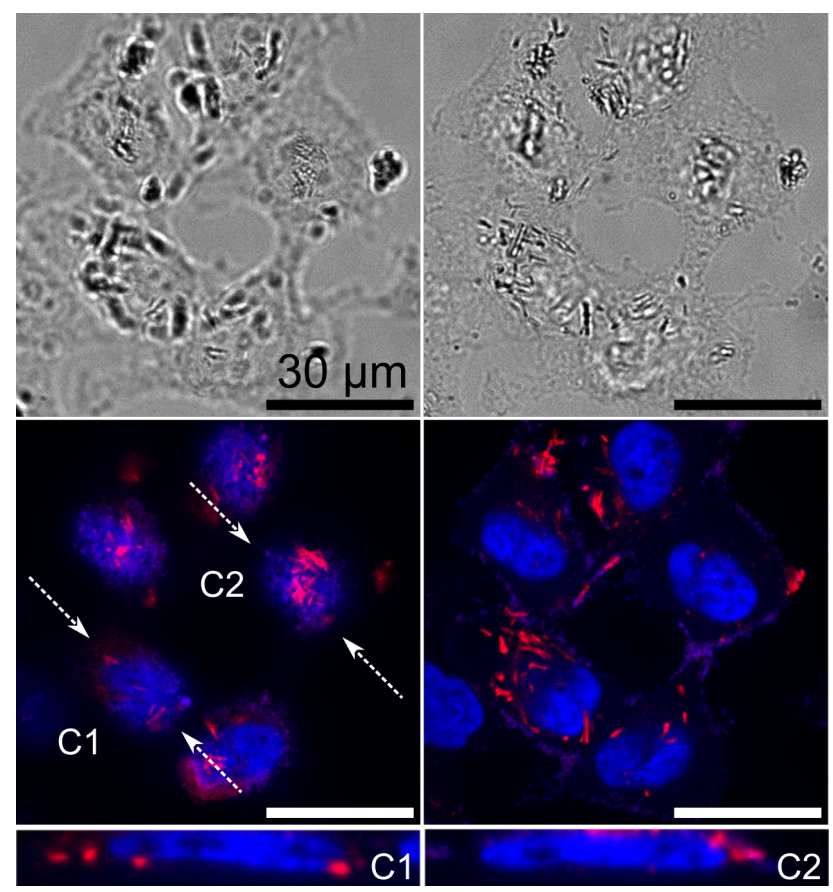

Figure 7: Bright field (upper row) and fluorescent (middle row) confocal images of HeLa cells incubated with bifunctional fluorescent magnetic $\mu$ NDs (60X objective). Reconstructed Zprofiles of cells $\mathbf{C} 1$ and $\mathbf{C 2}$ in the planes perpendicular to the highlighted regions of interest (lower row).

the cytosplam as the cell presents $\mu$ NDs at nucleus height within its walls. On cell $\mathbf{C 2}$ in left panel, many bright $\mu$ NDs appear on top of the out-o-focus nucleus, which disappear when diving at nucleus height. These $\mu$ NDs are most likely sitting on top of the cell or right on top of the nucleus within the cell walls. The Z-profile of cell C2 does not disambiguate the situation as such but with fluorescent labeling of the cell membrane would. At ay rate, the fluorescence feature of the $\mu \mathrm{NDs}$ enables quick assertion of the localisation of the probes to chose those to track to mechanically probe the rheological zones of interest.

\section{Summary}

In this work, bifunctional microneedles were readily assembled by electrostatic complexation of a ternary mixture of superparamagnetic iron oxide nanoparticles, red-emitting zinc-silverindium sulfide quantum dots and a cationic poylelectrolyte. The microneedles encompass 
the properties of both nanoparticulate components, namely superparamagnetism and fluorescence in the visible range. They can be actuated using small external magnetic fields and imaged using epifluorescence or confocal fluorescence microscopy. Interestingly, we found that by playing on the ternary composition, more specifically on the ratio of negative charges brought by the nanoparticles to positive charges brought by the polyelectrolyte, the length of the microneedles could be tuned in the 5-50 $\mu \mathrm{m}$ range. Because of their easy synthesis, their bifunctionality and their size tunability, these fluorescent and magnetic microneedles could find applications in multiple domains such as micromixing, bioassays or biomechanical studies and especially in the field of cell mechanics, since they were found to be readily internalised by model animal cells.

\section{Methods}

\subsection{Synthesis and characterisation of nanoparticle building blocks}

Maghemite mNPs were synthesized following the Massart procedure and surface-coated with sodium polyacrylate according to previously reported protocols [32,33]. PAA-coated ZAIS QDs were synthesized in one step following the method reported by Regulacio et al. with minor modifications [31]. Core material concentrations were determined by ICP-OES for QDs and X-ray fluorecence for mNPs. Concentrations of surface carboxylates were determined by $\mathrm{pH}$-titration with $\mathrm{HCl}$. The quantum yield of QDs was determined by comparison to those of fluorescein and rhodamine 6G [41-43]. Gel electrophoresis was performed on 1\%wt agarose gel in TBE $1 \mathrm{x}$ buffer. A full description of the synthetic and characterisation procedures is provided in Supporting Information.

\subsection{Assembly of fluorescent magnetic microneedles}

The following gives the synthetic procedure for assembly of $\{Z=4.20 ; w=0.20\}$ - $\mu$ NDs as an example. First, solutions of building blocks in $\mathrm{NH}_{4} \mathrm{Cl} 1 \mathrm{M} \mathrm{pH} 8$ were prepared by mixing in a 1:1 ratio a $\mathrm{NH}_{4} \mathrm{Cl} 2 \mathrm{M}$ pH8 solution with the $\mathrm{mNP}(0.2 \%$ wt core, $2.02 \mathrm{mM}$ carboxylate), the QD (0.4\% wt core, $14.73 \mathrm{mM}$ carboxylate) and polydiallyldimethylammonium chloride 
(0.2\% wt, $13.4 \mathrm{mM}$ ammonium) stock solutions respectively. Next, $400 \mu \mathrm{l}, 100 \mu \mathrm{l}$ and $40.5 \mu \mathrm{l}$ of $\mathrm{mNP}, \mathrm{QD}$ and polymer dilute solutions were mixed in an eppendorf tube and shaken for $10 \mathrm{~s}$ with the help of a vortex mixer. The ternary mixture was inserted in a $0.5-3 \mathrm{ml}$ Slide-aLyzer dialysis cassette with a $10 \mathrm{kDa}$ cutoff using a syringe. Air bubbles were removed. The cassette was nested into a custom made plexiglas sample holder sandwiched between two $5 \times$ $8 \mathrm{~cm}^{2} 0.3 \mathrm{~T}$ magnets. The sample holder was then immersed in a $2.5 \mathrm{~L}$ cylindrical flask filled with deionised water equipped with a stir bar and continuous monitoring of the conductivity was launched immediately. Dialysis was run for 2 hours after which the conductivity of the dialysis medium had reached a stable value. The content of the dialysis cassette had turned turbid, evidencing success of the assembly. The reaction media was carefully taken out using a syringe and inserted into a $2 \mathrm{ml}$ glass vial. $\mu$ NDs were sedimented using a soft table magnet and the supernatant was discarded. The $\mu$ NDs were redispersed in $1 \mathrm{ml}$ MilliQ water through gentle vortex mixing, sedimented again and the supernatant discarded. Two more washing steps using $2 \times 1 \mathrm{~mL}$ MilliQ water were performed. Finally, the $\mu$ NDs were redispersed in $1 \mathrm{~mL}$ MilliQ water and stored in the dark at $4^{\circ} \mathrm{C}$. The 14 other investigated compositions were prepared using the same protocol, with varying mNP, QD and polymer amounts, as given in Supporting Information.

\subsection{Imaging of $\mu$ NDs using phase contrast and epifluorescence mi- croscopy}

A square well was built on a glass slide using double-sided tape. The NW suspension was homogenized and diluted 4 times with MilliQ water. $8 \mu \mathrm{l}$ of this diluted suspension were deposited in the well, which was then covered with a coverslip. The sample was imaged in phase contrast mode and in epifluorescence mode using excitation and emission filters suited for Rhodamine 6G, with 10X, 20X and 40X magnifications. Length and brightness distributions were extracted using custom-written Matlab codes described in Supporting Information. 


\subsection{Imaging of fluorescent magnetic $\mu$ NDs in interaction with P32 HeLa cells using confocal fluorescence microscopy}

Preparation of NW sample. For incubation of cells, we selected $\{\mathrm{Z}=3.62, \mathrm{w}=0.15\}$ $\mu$ NDs. This sample had a mean length amongst the shortest in all investigated compositions $(15 \mu \mathrm{m})$. However, it was shown previously on a similar sized sample of magnetic-only $\mu$ NDs that cells operate a size sorting of $\mu$ NDs upon internalization, with an enrichment in $\mu$ NDs having a length around $4 \mu \mathrm{m}[29]$. We therefore shortened the $\mu$ NDs by ultrasonication before cell incubation. To this end, $400 \mu \mathrm{l}$ were inserted in a $1 \mathrm{~mm}$ optical path quartz cuvette and sonicated for 2 X 1 min in an ultrasonic bath in horizontal position, with intermediate cooling for $5 \mathrm{~min}$. The $\mu$ NDs were sedimented in the cuvette using a table magnet and the supernatant was discarded. Next, they were washed 3 times with $500 \mu$ l MilliQ water through sedimentation and discarding of the supernatant. Finally, they were dispersed in $500 \mu \mathrm{l}$ MilliQ water before autoclaving.

Cell culture. P32 HeLa cells were grown in T25-flasks as a monolayer in DMEM with high glucose $(4.5 \mathrm{~g} / \mathrm{L})$ and stable glutamine $(2 \mathrm{mM}$, PAA Laboratories GmbH, Austria). This medium was supplemented with $10 \%$ vt fetal bovine serum (FBS) and 1\% penicillin/streptomycin (PAA Laboratories GmbH, Austria), referred to as cell culture medium. Exponentially growing cultures were maintained in a humidified atmosphere of $5 \% \mathrm{CO}_{2}$ and $95 \%$ air at $37{ }^{\circ} \mathrm{C}$. In these conditions the plating efficiency was 70-90\% and the cell duplication time was $12-14 \mathrm{~h}$. Cell cultures were passaged twice weekly using trypsin-EDTA to detach the cells from their culture flasks and wells. For counting, the cells were pelleted by centrifugation at $1200 \mathrm{rpm}$ for $5 \mathrm{~min}$, supernatants were removed and cell pellets were resuspended in assay medium in Malassez counting chambers.

Seeding of P32 HeLa cells onto microscope glas coverslips. Sterilized microscope coverslips were nested in the wells of a 6 -well plate, washed with $1 \mathrm{~mL}$ of PBS buffer and then with $1 \mathrm{~mL}$ of culture medium. Cells were detached from their culture flask and transferred to the wells; the cell concentration was 75000 cells $/ \mathrm{ml}$ and each well contained $2 \mathrm{~mL}$ of cell suspension. The cells were incubated for 24 hours to promote cell adhesion onto the coverslips. 
Incubation of P32 HeLa cells with $\mu$ NDs. The cell medium was exchanged against $2 \mathrm{~mL}$ of PBS buffer and exchanged again for $1 \mathrm{~mL}$ of cuture medium. Next, 15, 30 or $40 \mathrm{muL}$ of shortened $\mu \mathrm{NDs}$ were mixed with $1 \mathrm{~mL}$ of culture medium and the resulting suspension was added dropwise in each well. The cells were incubated with the $\mu$ NDs for 20 hours after which they were washed by 2 rounds of medium exchange against PBS buffer.

Cell fixation. Next, the cells were fixed through incubation in 4\% PFA solution (J61899, Alfa Aesar) in PBS for 20 min at room temperature. After copious rinsing of PFA using PBS, the nuclei were fluorescently stained with DAPI dilactate dye ( $\geq 98 \%$, Aldrich) by incubation for $20 \mathrm{~min}$ at room temperature in a $2.2 \mu \mathrm{M}$ dye solution in PBS. The medium was exchanged for PBS three times to remove excess dye. Finally, microscope slides were equipped with Gene Frame stickers. The coverslips were carefully taken out of the wells and mounted onto the Gene Frames after filling the wells with PBS buffer. Samples were examined with an inverted wide-field microscope (Olympus IX81) equipped with spinning disc module (Yokogawa CSU-X1), with an oil immersion objective (60X, NA 1.42) and an EMCCD camera (Andor iXon 897). Z-stacks of wide-field fluorescent images were acquired using a piezo at $0.5 \mu \mathrm{m}$ increments. For the DAPI channel, $405 \mathrm{~nm}$ laser-line excitation and a $465 \mathrm{~nm}$-centered emission filter were used. For the NW chanel, the excitation line was 488 $\mathrm{nm}$ and the emission filter was centered at $607 \mathrm{~nm}$.

\section{References}

[1] Randall M. Erb, Rafael Libanori, Nuria Rothfuchs, and AndrÃl R. Studart. Composites Reinforced in Three Dimensions by Using Low Magnetic Fields. Science, 335(6065):199204, January 2012.

[2] Kevin Ward and Z. Hugh Fan. Mixing in microfluidic devices and enhancement methods. Journal of Micromechanics and Microengineering, 25(9):094001, 2015.

[3] Wen Han Chong, Lip Ket Chin, Rachel Lee Siew Tan, Hong Wang, Ai Qun Liu, and Hongyu Chen. Stirring in Suspension: Nanometer-Sized Magnetic Stir Bars. Angewandte Chemie International Edition, 52(33):8570-8573, August 2013. 
[4] Dirk De Bruyker, Michael I. Recht, Ali Asgar S. Bhagat, Francisco E. Torres, Alan G. Bell, and Richard H. Bruce. Rapid mixing of sub-microlitre drops by magnetic microstirring. Lab on a Chip, 11(19):3313-3319, September 2011.

[5] Kee Suk Ryu, Kashan Shaikh, Edgar Goluch, Zhifang Fan, and Chang Liu. Micro magnetic stir-bar mixer integrated with parylene microfluidic channels. Lab on a Chip, 4(6):608-613, November 2004.

[6] Sibani Lisa Biswal and Alice P. Gast. Micromixing with Linked Chains of Paramagnetic Particles. Analytical Chemistry, 76(21):6448-6455, November 2004.

[7] Hao Yu, Thien-Binh Nguyen, Sum Huan Ng, and Tuan Tran. Mixing control by frequency variable magnetic micropillar. RSC Advances, 6(14):11822-11828, January 2016.

[8] Ahmed Alfadhel and JÃijrgen Kosel. Magnetic Nanocomposite Cilia Tactile Sensor. Advanced Materials, 27(47):7888-7892, December 2015.

[9] A. Alfadhel, B. Li, A. Zaher, O. Yassine, and J. Kosel. A magnetic nanocomposite for biomimetic flow sensing. Lab on a Chip, 14(22):4362-4369, October 2014.

[10] Yibo Gao, Bingpu Zhou, Xiaoxiao Wu, Xinghua Gao, Xiping Zeng, Jiao Xie, Cong Wang, Ziran Ye, Jun Wan, and Weijia Wen. Three Dimensional and Homogenous Single Cell Cyclic Stretch within a Magnetic Micropillar Array (mMPA) for a Cell Proliferation Study. Acs Biomaterials Science 65 Engineering, 2(1):65-72, January 2016. WOS:000369348700007.

[11] Jimmy le Digabel, Nicolas Biais, JÃlrome Fresnais, Jean-FranÃ ğois Berret, Pascal Hersen, and Benoit Ladoux. Magnetic micropillars as a tool to govern substrate deformations. Lab on a Chip, 11(15):2630-2636, July 2011.

[12] Nathan J. Sniadecki, Corinne M. Lamb, Yaohua Liu, Christopher S. Chen, and Daniel H. Reich. Magnetic microposts for mechanical stimulation of biological cells: Fabrication, characterization, and analysis. Review of Scientific Instruments, 79(4):044302, April 2008. 
[13] J.-F. Berret. Local viscoelasticity of living cells measured by rotational magnetic spectroscopy. Nature Communications, 7:10134, January 2016.

[14] L. Chevry, N. K. Sampathkumar, A. Cebers, and J.-F. Berret. Magnetic wire-based sensors for the microrheology of complex fluids. Phys. Rev. E, 88(6):062306, December 2013.

[15] Denis Wirtz. Particle-Tracking Microrheology of Living Cells: Principles and Applications. Annual Review of Biophysics, 38(1):301-326, 2009.

[16] T. A. Waigh. Microrheology of complex fluids. Reports on Progress in Physics, 68(3):685, March 2005.

[17] Houcine Labiadh, Tahar Ben Chaabane, Romain Sibille, Lavinia Balan, and Raphã̃nl Schneider. A facile method for the preparation of bifunctional Mn:ZnS/ZnS/Fe ${ }_{3} \mathrm{O}_{4}$ magnetic and fluorescent nanocrystals. Beilstein Journal of Nanotechnology, 6:17431751, August 2015.

[18] Peng Jing, Qin Wang, Baocang Liu, Guangran Xu, Yanbing Zhang, Jun Zhang, and Gejihu De. Controlled fabrication of bi-functional Fe3o4@SiO2@Gd2o3:Yb,Er nanoparticles and their magnetic, up-conversion luminescent properties. RSC Advances, 4(84):44575-44582, September 2014.

[19] Yanjiao Lu, Yang Zheng, Shusen You, Feng Wang, Zhuo Gao, Jie Shen, Wantai Yang, and Meizhen Yin. Bifunctional Magnetic-Fluorescent Nanoparticles: Synthesis, Characterization, and Cell Imaging. ACS Applied Materials \& Interfaces, 7(9):5226-5232, March 2015.

[20] Natalia Hassan, ValÂlrie Cabuil, and Ali Abou-Hassan. Continuous Multistep Microfluidic Assisted Assembly of Fluorescent, Plasmonic, and Magnetic Nanostructures. Angewandte Chemie International Edition, 52(7):1994-1997, February 2013.

[21] Juan Peng, Li-Na Feng, Kui Zhang, Jing-Jing Li, Li-Ping Jiang, and Jun-Jie Zhu. Multifunctional Manganese Carbonate Microspheres with Superparamagnetic and Flu- 
orescent Properties: Synthesis and Biological Application. Chemistry ăĂS A European Journal, 17(39):10916-10923, September 2011.

[22] Tobias Bollhorst, Shakiba Shahabi, Katharina WÃürz, Charlotte Petters, Ralf Dringen, Michael Maas, and Kurosch Rezwan. Bifunctional Submicron Colloidosomes Coassembled from Fluorescent and Superparamagnetic Nanoparticles. Angewandte Chemie International Edition, 54(1):118-123, January 2015.

[23] Xiaoyu Sun, Ke Ding, Yi Hou, Zhenyu Gao, Wensheng Yang, Lihong Jing, and Mingyuan Gao. Bifunctional Superparticles Achieved by Assembling Fluorescent CuInS2@ZnS Quantum Dots and Amphibious Fe3o4 Nanocrystals. The Journal of Physical Chemistry C, 117(40):21014-21020, October 2013.

[24] Riccardo Di Corato, Nadja C. Bigall, Andrea Ragusa, Dirk Dorfs, Alessandro Genovese, Roberto Marotta, Liberato Manna, and Teresa Pellegrino. Multifunctional Nanobeads Based on Quantum Dots and Magnetic Nanoparticles: Synthesis and Cancer Cell Targeting and Sorting. Acs Nano, 5(2):1109-1121, February 2011. WOS:000287553800049.

[25] Hui Wang, Jinhui Yi, Sumit Mukherjee, Probal Banerjee, and Shuiqin Zhou. Magnetic/NIR-thermally responsive hybrid nanogels for optical temperature sensing, tumor cell imaging and triggered drug release. Nanoscale, 6(21):13001-13011, October 2014.

[26] Bipin Kumar Gupta, Satbir Singh, Pawan Kumar, Yean Lee, Garima Kedawat, Tharangattu N. Narayanan, Sajna Antony Vithayathil, Liehui Ge, Xiaobo Zhan, Sarika Gupta, Angel A. MartÃy, Robert Vajtai, Pulickel M. Ajayan, and Benny Abraham Kaipparettu. Bifunctional Luminomagnetic Rare-Earth Nanorods for High-Contrast Bioimaging Nanoprobes. Scientific Reports, 6:32401, September 2016.

[27] J. Fresnais, J.-F. Berret, B. Frka-Petesic, O. Sandre, and R. Perzynski. Electrostatic CoAssembly of Iron Oxide Nanoparticles and Polymers: Towards the Generation of Highly Persistent Superparamagnetic Nanorods. Advanced Materials, 20(20):3877-3881, 2008. 
[28] M. Yan, J. Fresnais, and J.-F. Berret. Growth mechanism of nanostructured superparamagnetic rods obtained by electrostatic co-assembly. Soft Matter, 6(9):1997, 2010.

[29] Malak Safi, Minhao Yan, Marie-Alice Guedeau-Boudeville, HÃllấlne Conjeaud, Virginie Garnier-Thibaud, Nicole Boggetto, Armelle Baeza-Squiban, Florence Niedergang, Di-

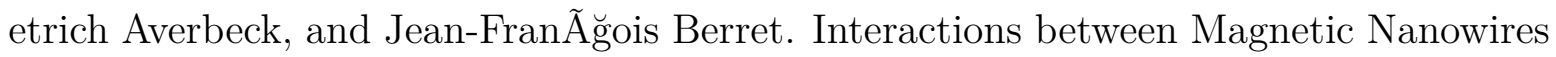
and Living Cells: Uptake, Toxicity, and Degradation. ACS Nano, 5(7):5354-5364, July 2011.

[30] M. Yan, J. Fresnais, S. Sekar, J.-P. Chapel, and J.-F. Berret. Magnetic Nanowires Generated via the Waterborne Desalting Transition Pathway. ACS Appl. Mater. Interfaces, 3(4):1049-1054, April 2011.

[31] Michelle D. Regulacio, Khin Yin Win, Seong Loong Lo, Shuang-Yuan Zhang, Xinhai Zhang, Shu Wang, Ming-Yong Han, and Yuangang Zheng. Aqueous synthesis of highly luminescent AgInS2âĂŞZnS quantum dots and their biological applications. Nanoscale, 5(6):2322-2327, March 2013.

[32] R. Massart, E. Dubois, V. Cabuil, and E. Hasmonay. Preparation and Properties of Monodisperse Magnetic Fluids. J. Magn. Magn. Mater., 149(1-2):1-5, August 1995. WOS:A1995RR75100002.

[33] Ling Qi, Jean-Paul Chapel, Jean-Christophe Castaing, JÃlrãtme Fresnais, and JeanFranÃ ğois Berret. Stability and Adsorption Properties of Electrostatic Complexes:âĂL Design of Hybrid Nanostructures for Coating Applications. Langmuir, 23(24):1199611998, November 2007.

[34] Prasad Subramaniam, Seung Jae Lee, Shreyas Shah, Sahishnu Patel, Valentin Starovoytov, and Ki-Bum Lee. Generation of a Library of Non-Toxic Quantum Dots for Cellular Imaging and siRNA Delivery. Advanced Materials, 24(29):4014-4019, 2012.

[35] Gaëlle Charron, Dominik Hühn, AurÃllie Perrier, Laure Cordier, Christopher J. Pickett, Thomas Nann, and Wolfgang J. Parak. On the Use of pH Titration to Quantitatively Characterize Colloidal Nanoparticles. Langmuir, 28(43):15141-15149, October 2012. 
[36] Richard O. Duda and Peter E. Hart. Use of the Hough Transformation to Detect Lines and Curves in Pictures. Commun. ACM, 15(1):11-15, January 1972.

[37] Riccardo Di Corato, Philomena Piacenza, Mariarosaria MusarÃš, Raffaella Buonsanti, Pantaleo Davide Cozzoli, Massimo Zambianchi, Giovanna Barbarella, Roberto Cingolani, Liberato Manna, and Teresa Pellegrino. MagneticâĂŞFluorescent Colloidal Nanobeads: Preparation and Exploitation in Cell Separation Experiments. Macromolecular Bioscience, 9(10):952-958, October 2009.

[38] Irene Sinn, Theodore Albertson, Paivo Kinnunen, David N. Breslauer, Brandon H. McNaughton, Mark A. Burns, and Raoul Kopelman. Asynchronous Magnetic Bead Rotation Microviscometer for Rapid, Sensitive, and Label-Free Studies of Bacterial Growth and Drug Sensitivity. Analytical Chemistry, 84(12):5250-5256, June 2012. WOS:000305320500015.

[39] Vinay Swaminathan, Karthikeyan Mythreye, E. Tim O’Brien, Andrew Berchuck, Gerard C. Blobe, and Richard Superfine. Mechanical stiffness grades metastatic potential in patient tumor cells and in cancer cell lines. Cancer Res, page canres.0247.2011, June 2011.

[40] Thomas Grevesse, Borna E. Dabiri, Kevin Kit Parker, and Sylvain Gabriele. Opposite rheological properties of neuronal microcompartments predict axonal vulnerability in brain injury. Scientific Reports, 5:9475, March 2015.

[41] Albert M. Brouwer. Standards for photoluminescence quantum yield measurements in solution (IUPAC Technical Report). Pure and Applied Chemistry, 83(12):2213-2228, August 2011.

[42] M. Fischer and J. Georges. Fluorescence quantum yield of rhodamine $6 \mathrm{~g}$ in ethanol as a function of concentration using thermal lens spectrometry. Chemical Physics Letters, 260(1):115-118, September 1996. 
[43] Laurent Porrès, Adam Holland, Lars-Olof På lsson, Andrew P. Monkman, Chris Kemp, and Andrew Beeby. Absolute Measurements of Photoluminescence Quantum Yields of Solutions Using an Integrating Sphere. J Fluoresc, 16(2):267-273, February 2006. 